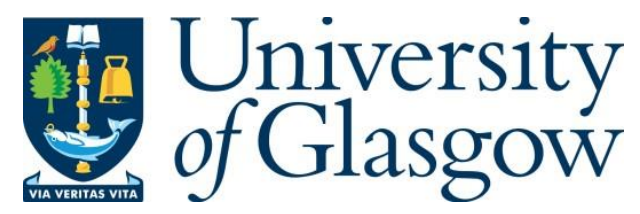

Schmitz, L., Gambichler, T., Kost, C., Gupta, G., Stücker, M., Stockfleth, E. and Dirschka, T. (2018) Cutaneous squamous cell carcinomas are associated with basal proliferating actinic keratoses. British Journal of Dermatology, (doi:10.1111/bjd.16536).

There may be differences between this version and the published version. You are advised to consult the publisher's version if you wish to cite from it.

This is the peer reviewed version of the following article Schmitz, L., Gambichler, T., Kost, C., Gupta, G., Stücker, M., Stockfleth, E. and Dirschka, T. (2018)

Cutaneous squamous cell carcinomas are associated with basal proliferating actinic keratoses. British Journal of Dermatology, which has been published in final form at http://dx.doi.org/10.1111/bjd.16536. This article may be used for non-

commercial purposes in accordance with Wiley Terms and Conditions for SelfArchiving.

http://eprints.gla.ac.uk/159091/

Deposited on: 27 March 2018

Enlighten - Research publications by members of the University of Glasgow http://eprints.gla.ac.uk 
DR LUTZ SCHMITZ (Orcid ID : 0000-0002-9627-2179)

DR THILO GAMBICHLER (Orcid ID : 0000-0001-7862-3695)

Article type : Original Article

\title{
Cutaneous squamous cell carcinomas are associated with basal proliferating actinic keratoses
}

\author{
L. Schmitz ${ }^{1 *}$, T. Gambichler ${ }^{1}$, C. Kost ${ }^{1}$, G. Gupta ${ }^{2,3}$, M. Stücker ${ }^{1}$, E. Stockfleth ${ }^{1}$, T. \\ Dirschka $^{4,5}$ \\ 1Department of Dermatology, Venereology and Allergology, Ruhr-University, Bochum, Germany \\ 2Department of Dermatology, University Hospital Monklands, Lanarkshire, UK \\ 3School of Medicine, University of Glasgow, UK \\ 4CentroDerm Clinic, Heinz-Fangman-Straße 57, Wuppertal, Germany \\ 5Faculty of Health, University Witten-Herdecke, Alfred-Herrhausen-Straße 50, Witten, Germany
}

Running head: iSCCs are associated with basal proliferating AKs

IRB Approval: obtained

Funding source: None

Keywords: actinic keratosis, basal growth, squamous cell carcinoma, protruding, PRO

This article has been accepted for publication and undergone full peer review but has not been through the copyediting, typesetting, pagination and proofreading process, which may lead to differences between this version and the Version of Record. Please cite this article as doi: $10.1111 /$ bjd. 16536

This article is protected by copyright. All rights reserved. 
*Corresponding author: Dr. Lutz Schmitz

Address: Department of Dermatology, Ruhr-University, Gudrunstr. 56, D-44791

Bochum, Germany

Phone: +49 (0)234 5090

Fax: +49 (0)2345093445

Email: I.schmitz@klinikum-bochum.de

\section{WHAT'S ALREADY KNOWN ABOUT THIS TOPIC?}

- Actinic keratosis (AK) can progress into invasive squamous cell carcinoma (iSCC)

- The established histological classification of AKs lacks predictive properties to determine progression risk

- A recently proposed classification scheme has reported on the histological changes in the epidermis particularly the downward directed proliferation of atypical keratinocytes at the basal layer

\section{WHAT DOES THIS STUDY ADD?}

- Invasive squamous cell carcinomas (iSCC) are associated with basal proliferation of actinic keratoses (AKs)

- In our study, AK I is confirmed to be the dominant AK grade adjacent to iSCC

- ISCCs are more commonly associated with marked basal proliferation (PRO III) and with adnexal involvement, along with atypical keratinocytes restricted to the lower third of the epidermis (AK I) 


\section{ABSTRACT}

Background In addition to the extent of atypical keratinocytes throughout the epidermis, actinic keratoses (AKs) are histologically characterized by downward directed basal layer expansion. It is not known if this growth pattern correlates with the risk of developing invasive squamous cell carcinoma (iSCC).

Objective. To characterize the prevalence of downward directed basal layer expansion of AKs adjacent to iSCC.

Methods The epidermis overlying and adjacent to iSCCs was assessed histologically. We determined the histological grade (AKI-III), basal growth pattern (PROI-III) and accompanying parameters such as adnexal involvement.

Results Of 307 lesions, $52.4 \%$ of AKs were histologically classified as AKI, $38.1 \%$ as AKII, and $6.8 \%$ as AKIII (chi-squared; $\mathrm{P}<0.0001$ ). $2.6 \%$ of adjacent epidermis did not show any atypical keratinocytes. The epidermis adjacent to iSCCs was classified as having a PROI basal growth pattern in $25.7 \%$, PROII in $31.9 \%$, and PROIII in $39.4 \%$ cases. $2.9 \%$ of AKs showed no basal growth (chi-squared; $\mathrm{P}<0.0001$ ).

$118(48.8 \%)$ AKs showed extension into adnexal structures. These AKs were graded as PROI in $18.6 \%$, PROII in $30.5 \%$, and PROIII in 50.8\%. The epidermis above iSCCs could only be assessed for upwards directed growth and showed no significant differences in the three AK grades $(P=0.4211)$.

This article is protected by copyright. All rights reserved. 
Conclusions Basal proliferative AKs as well as atypical keratinocytes restricted to the lower third of the epidermis are most commonly seen adjacent to iSCC with less evidence for full thickness epidermal dysplasia. Our study supports the important role of dysplastic keratinocytes in the epidermal basal layer and their potential association with iSCC.

\section{INTRODUCTION}

Actinic keratoses (AKs) are generally considered to be low-risk disease with in-situ keratinocyte dysplasia. However, AKs can progress into invasive squamous cell carcinoma (iSCC) and subsequently metastasize. ${ }^{1-4}$ The risk of progression of distinct AKs to iSCC ranges between $0.025 \%$ and $16 \%$ per year. ${ }^{5}$ AKs are a common diagnosis in dermatological practice and increasing incidence of AKs is observed due to a steadily ageing population in industrialised countries. The overall prevalence of AKs is estimated to range between 6 and $26 \%$. $^{3,6}$ Geographical regions with high ultraviolet (UV) exposure such as Australia present even higher prevalences (40 to $60 \%)^{7}$

AKs are usually diagnosed clinically. Histologically, AKs are characterized by the extent of atypical keratinocytes throughout the epidermal layer. An established grading scheme classifies atypical keratinocytes within the lower third of the epidermis as AK I, atypical keratinocytes within the lower two thirds as AK II, and full thickness involvement of atypical keratinocytes as AK III or in-situ carcinoma. ${ }^{2}$ Two recent studies have shown that the clinical appearance of AK does not match with the underlying grade of histological dysplasia. ${ }^{8,9}$ In analogy to cervical intraepithelial neoplasia $(\mathrm{CIN})$, the extent of epidermal dysplasia was thought to be part of a 
disease continuum in a multi-step carcinogenesis process. ${ }^{10}$ However, FernandezFigueras et al. demonstrated that AKs with atypical keratinocytes restricted to the lower third of the epidermis (AKI) were most commonly associated with iSCC. ${ }^{11}$ This suggests that upward extension of dysplastic cells may have less prognostic significance and AKs independent of their histological grading (AK I-III) could progress into iSCC which in the absence of longitudinal studies may, along with the actinically damaged field, require treatment.

However, this strategy may lead to overtreatment in many cases with its associated costs, particularly as numbers of patients continue to increase. Therefore, other classifications or scores for risk stratification of AKs are required. A recently proposed histological classification reported on the basal growth patterns of AKs. ${ }^{12}$ Assuming that dermal invasion starts in the direct vicinity of the basement membrane zone, this classification differentiates basal growth patterns into three grades of atypical keratinocytes protruding into the dermis.

As all existing clinical and histological classifications lack predictive value, it was the aim of this study to investigate a possible relationship between basal growth patterns of AKs adjacent to iSCC. To the best of our knowledge, this is the first study which provides a systematic analysis of both upwards-directed growth (AK I-III) ${ }^{2}$ and downwards-directed growth (PRO I-III) ${ }^{12}$ patterns and their association with iSCC.

This article is protected by copyright. All rights reserved. 


\section{MATERIALS AND METHODS}

\section{Study population}

This retrospective study was conducted according to the Declaration of Helsinki and performed at the Skin Cancer Center of the Ruhr-University Bochum (Bochum, Germany). The study was approved by the ethics review board of the RuhrUniversity Bochum. Our database was consecutively searched from January 2015 to March 2017 for histologically diagnosed iSCCs located on the head. We restricted this study to the head to ensure regional homogenicity for comparison. Collision tumours and samples from immunosuppressed patients were excluded from this study. Overall, 479 tumours were identified and were eligible for histological analysis.

\section{Microscopic evaluation}

Prior to classification, all haematoxylin and eosin (H\&E) stained sections (4 $\mu \mathrm{m}$ of thickness) of each tumour were analysed to identify an adequate section showing transition between in-situ and invasive parts of SCC. Only primary iSCCs were included and therefore tumours with only a dermal component were excluded from this study. SCCs with histological features of a keratoacanthoma and recurrent tumours were also excluded. Subsequently, the epidermis adjacent to and overlying the invasive areas in the selected sections $(n=307)$ was investigated by two independent investigators (LS and TD). Disagreements between the two investigators were discussed and resolved at a double-headed microscope. 
Basal growth pattern of AKs was determined exclusively in the direct vicinity of the iSCC and recorded as showing crowding of basal atypical keratinocytes (PRO I), slightly budding of atypical keratinocytes into the upper papillary dermis and forming round nests of atypical keratinocytes (PRO II), or spiky or filiform papillary elongation of atypical keratinocytes protruding into upper dermis and exceeding the thickness of the overlying epidermis (PRO III, papillary sprouting). ${ }^{12}$ The epidermis overlying iSCCs was not evaluated for basal proliferation as downward-directed proliferation is already a characteristic feature of invasive tumour.

Upwards-directed growth pattern according to Röwert-Huber et al. was evaluated at the adjacent epidermis and at the epidermis overlying iSCCs. In grade AK I, atypical keratinocytes are restricted to the basal and suprabasal layers, limited to the lower third of the epidermis. In grade AK II, atypical keratinocytes extend to the lower two thirds of the epidermis. In grade AK III, full thickness atypia of the epidermis is found regarded as in-situ SCC. ${ }^{2}$ The overlying epidermis of iSCCs with ulceration of more than $50 \%$ in diameter was not classified as this would distort the histological grade of AK. ${ }^{11}$ In cases of different grades in both classifications, the highest grade was chosen to classify the lesion.

In addition, involvement of adnexal structures adjacent to iSCCs were evaluated. Involvement was defined as positive when atypical keratinocytes extended along adnexal structures exceeding the thickness of the overlying epidermis. 


\section{Statistical analysis}

Data analysis was performed using the statistical package MedCalc software version 17.8.6 (Ostend, Belgium). The distribution of data was assessed by the D’AgostinoPearson test. If there was normal distribution, data were expressed as mean and standard deviation (SD); if not, data were expressed as median and range. Data were statistically analyzed using the Chi-squared test, with P-values less than 0.05 being considered statistically significant.

\section{RESULTS}

In total, 307 iSCCs were included in this study. The study population had a median (interquartile range; IQR) age of 81 years $(76-86$ years) and most patients were male (73.6\%). $59.6 \%$ had a history of at least one iSCC prior to this study. The majority of tumours were located on the face (72.3\%) and showed a median (IQR) tumour thickness of $1.9 \mathrm{~mm}(1.1-3.9 \mathrm{~mm})$. Most of them were well differentiated (85.3\%) and invaded into the reticular dermis (75.9\%). (Table 1)

The epidermis adjacent to iSCCs exhibited no signs of actinic damage in $2.6 \%$ of cases. The remaining number of cases with AK I, AK II and AK III changes adjacent to SCC were $161(52.4 \%), 117(38.1 \%)$, and $21(6.8 \%)$, respectively (chi-squared; P $<0.0001)$. Regarding the basal growth pattern, 9 (2.9\%) showed no basal growth, 79 (25.7\%) were classified as PRO I, 98 (31.9\%) as PRO II, and 121 (39.4\%) as PRO III (chi-squared; $\mathrm{P}<0.0001$ ). (Table 2) No significant correlation (chi-square, $\mathrm{P}=$ 0.1894) between histological classification (AK I-III) and underlying basal growth pattern (PRO I-III) of adjacent AKs was demonstrated. (Fig. 1a) 
Overall, adnexal structures were present in 242 samples (78.8\%). There was involvement of adnexal structures in 118 cases $(48.8 \%)$ and this was more frequently observed in adjacent AKs graded as AK I (59/118, 50\%) compared to AK II $(53 / 118,44.9 \%)$ and AK III $(6 / 118,5.1 \%)$. In contrast, basal growth patterns in cases with affected adnexal structures, showed PRO I change in 22/118 (18.6\%), followed by PRO II in 36/118(30.5\%), and PRO III in 60/118(50.8\%). The distribution of gradings was significantly different throughout the PRO and AK classifications $(P<0.0001)$. (Fig. 2)

With regards to the evaluation of the epidermis overlying iSCCs, 85 cases $(27.7 \%)$ were excluded due to horizontal ulceration of more than $50 \%$. Mann-Whitney-U test showed a significant difference in tumour thickness of lesions with ulceration of more than $50 \%$ and less than $50 \%(P=0.0006)$. Of the remaining 222 iSCCs, $76(34.2 \%)$ were classified as AK I, 69 (31.1\%) as AK II and 77 (34.7\%) as AK III. (Fig. 1b) Foci of ulceration were present in 74 cases (32.9\%), of which 22 corresponded to lesions with AK I in overlying epidermal layers (29.7\%), 22 with AK II (29.7.6\%) and 30 with AK III (40.5\%). No significant difference in the distribution of histological grades was observed $(P=0.4211)$.

This article is protected by copyright. All rights reserved. 


\section{DISCUSSION}

Our study showed that AKs presenting with advanced basal growth patterns (PRO II and III) are most commonly found adjacent to iSCCs. Moreover, this study shows that AKs with atypical keratinocytes restricted to the lower third of the epidermis (AK I) are the most frequent grading adjacent to invasive tumour supporting the findings of a previous study. ${ }^{11}$ When findings of both classification schemes are combined, basal atypical keratinocytes seem to play an important role in tumour progression.

The ability of cells to invade is characterized by their propensity to pass through the basement membrane and extracellular matrix. ${ }^{13,14}$ Thus, atypical keratinocytes next to the basement membrane bear the highest potential to penetrate into underlying dermal tissues. Additionally, every AK is defined by atypical keratinocytes at the basal layer which in turn migrate upwards in the multistep carcinogenesis concept. $^{2,15}$ There seems to be no rational explanation as to why atypical cells should first extend through the whole epidermis until they acquire the ability to pass through the basement membrane. Recent approaches show that the concept of epithelial-to-mesenchymal transition (EMT), which is an established theory in cancer

research, plays an important role in terms of progression. ${ }^{16}$ The extracellular matrix hosts multiple cell populations including fibroblasts, pericytes, endothelial cells, cytokines and immune regulatory cells. ${ }^{17}$ On the one hand, atypical keratinocytes may be restricted or controlled by stromal tissue and only have the opportunity to grow upwards (AK II-III) presenting a low risk of progression. However, it is likely that stromal tissue is not able to control atypical keratinocytes adequately allowing basal layer protrusion into dermal tissue. Subsequently, atypical keratinocytes may pass the basement membrane and therefore these downward projections (PRO II-III) may 
represent a higher progression risk. (Fig. 3a) Our study confirms a significant correlation with iSCCs. Cancer-associated fibroblasts (CAFs) promote cancer cell invasion through the basement membrane and it has been shown that CAFs facilitate the breaching of the basement membrane in a matrix metalloproteinaseindependent manner. ${ }^{18}$ This important role of CAFs has been demonstrated in peritumoral skin of basal cell carcinoma. ${ }^{19}$

Histological classification systems should discriminate morphological features to ideally stratify progression risk. From this point of view, classifying atypical keratinocytes adjacent to the basement membrane could offer a better risk stratification than evaluating the whole epidermal layer. Basal proliferation leads to an enlargement of basement membrane which provides a larger contact zone between dysplastic cells and basement membrane. Moreover, the proliferation of atypical keratinocytes and subsequent protrusion into upper dermal layers may be a non-invasive precursor. This may resemble a growth pattern of invasive cells which do not yet have the ability to penetrate the basement membrane.

This study exclusively assessed histological sections of iSCC containing the junction between invasive tumour tissue and non-invasive epidermis. Adjacent or overlying epidermis without any connection to underlying tumour tissue could not be assessed with regard to a clear relationship. Without a visible connection to epidermal layers tumour tissue may have undergrown healthy epidermis and therefore could not be reliably evaluated. In this context, the question of progression from contiguous tissue to invasive carcinoma emerges. If tumour development is regarded as a single event which spreads centrifugally, overlying and adjacent tissue may not provide 
information on distinct morphological factors which may have led to tumour genesis. Nevertheless, morphological properties of tumour environment or field cancerisation characterized by histological classifications may indicate factors with higher risk of carcinogenesis. Thus, this study exhibits basal growth pattern to be important in terms of progression risk.

Our study showed no differences in grading of AKs overlying iSCC. These results contrast the investigation of Fernandez-Figueras et al., who found AK I to be the most common lesion above iSCC. ${ }^{11}$ These differences might be explained by different study populations. Our study population was evaluated for overlying epidermis only in slides presenting with an apparent transition between AK and iSCC. We excluded those slides in which there was no connection between tumour cells and the overlying epidermis. Moreover, it might also result from the evaluation of the epidermis next to areas of ulceration. The closer the assessment to ulceration is performed, the more frequent full thickness atypia may be seen leading to higher AK grade classification.

This study assessed the most common grading for lesions with adnexal involvement (Fig. 3b) involving both the upwards-directed classification (AK I-III), and the downwards-directed classification (PRO I-III) ${ }^{12}$. Both classifications illustrate the importance of basal proliferation and adnexal involvement of atypical cells. These finding are supported by two other studies exhibiting AK I and PRO III to be the most frequent classifications related to adnexal structure involvement. ${ }^{11,12}$ Therefore it is unlikely that atypia in upper epidermal layers plays a role in basal proliferation of AK. 
With regard to recurrence rates following different treatment approaches, adnexal involvement and the distinct basal proliferation seems to be especially important.

Overall, current clinical and histological classification systems of AK do not allow a reliable risk stratification of patients enabling physicians to make adequate treatment decisions. This study indicates the importance of basal growth pattern of AKs in terms of risk stratification in contrast to the established classification scheme. Needless to say that AK II and AK III can become invasive, but we are not able to detect if this shift is caused by atypical keratinocytes which have finally extended throughout the whole epidermis or by basal keratinocytes. A possible explanation could be that AKs which do not progress at early stages (according to Röwert-Huber et al. $^{2}$ ) into invasive carcinoma do not offer a high risk disposition to penetrate the basement membrane and interact with or are controlled by extracellular matrix. Thus, atypical cells may remain restricted to epidermal layers and extend in epidermal tissue. The established classification scheme ${ }^{2}$ seems not to be reliable for risk stratification and focuses on a merely morphological description without any predictive value.

A limitation of our study was that adjacent and overlying AKs were assessed, but we were not able to evaluate the original AK the iSCC had originated from. Changes at mutational level most probably lead to the final progression of this distinct subtype of AK. In conclusion, basal proliferation classified by its morphological pattern and involvement of adnexal structures was significantly associated with iSCC. Thus, histological evaluation of AKs should also report on the basal growth pattern (PROgrading). Besides these morphological criteria, further studies should focus on stromal cells such as CAFs and their role in the information exchange between epidermal and dermal tissue. 


\section{ACKNOWLEDGEMENT}

This work is part of the doctoral thesis of Christian Kost

\section{Conflict of interest disclosures:}

L. Schmitz: Lecture fees from Almirall, Biofrontera, Galderma, Meda, Riemser

T. Gambichler: Lecture fees from Novartis, Roche, NeraCare, BMS.

C. Kost: None.

G. Gupta: Lecture fees from Abbvie, Galderma, Leo and Meda. Member of advisory boards for Almirall, Leo, Meda and Novartis.

M. Stücker: Lecture fees from Juzo, Medi; member of advisory boards for Eurocom, Bauerfeind

E. Stockfleth: Lectures from Almirall, Leo, Pierre Favre, Philips

T. Dirschka: Lecture fees from Almirall, Biofrontera, Galderma, Hexal, Leo, Meda, Riemser, Janssen; member of advisory boards for Almirall, Biofrontera, Leo, Meda, Novartis, Riemser, Janssen; unrestricted grants from Meda and Galderma

\section{REFERENCES}

1 Ackerman AB, Mones JM. Solar (actinic) keratosis is squamous cell carcinoma. Br J Dermatol 2006; 155: 9-22.

2 Rowert-Huber J, Patel MJ, Forschner T et al. Actinic keratosis is an early in situ squamous cell carcinoma: a proposal for reclassification. Br J Dermatol 2007; 156 Suppl 3: 8-12.

3 Salasche SJ. Epidemiology of actinic keratoses and squamous cell carcinoma. J Am Acad Dermatol 2000; 42: 4-7. 
4 Stockfleth E, Ferrandiz C, Grob JJ et al. Development of a treatment algorithm for actinic keratoses: a European Consensus. Eur J Dermatol 2008; 18: 651-9.

5 Glogau RG. The risk of progression to invasive disease. J Am Acad Dermatol 2000; 42: 23-4.

6 Memon AA, Tomenson JA, Bothwell J et al. Prevalence of solar damage and actinic keratosis in a Merseyside population. Br J Dermatol 2000; 142: 11549.

7 Frost C, Williams G, Green A. High incidence and regression rates of solar keratoses in a queensland community. J Invest Dermatol 2000; 115: 273-7.

8 Heerfordt IM, Nissen CV, Poulsen T et al. Thickness of Actinic Keratosis Does Not Predict Dysplasia Severity or P53 Expression. Sci Rep 2016; 6: 33952.

9 Schmitz L, Kahl P, Majores M et al. Actinic keratosis: correlation between clinical and histological classification systems. J Eur Acad Dermatol Venereol 2016; 30: 1303-7.

10 Quatresooz P, Pierard-Franchimont C, Paquet P et al. Crossroads between actinic keratosis and squamous cell carcinoma, and novel pharmacological issues. Eur J Dermatol 2008; 18: 6-10.

11 Fernandez-Figueras MT, Carrato C, Saenz X et al. Actinic keratosis with atypical basal cells $(\mathrm{AKI})$ is the most common lesion associated with invasive squamous cell carcinoma of the skin. J Eur Acad Dermatol Venereol 2015;

29: 991-7.

12 Schmitz L, Gambichler T, Gupta G et al. Actinic keratoses show variable histological basal growth patterns - a proposed classification adjustment. $J$ Eur Acad Dermatol Venereol 2017. 
13 Clark AG, Vignjevic DM. Modes of cancer cell invasion and the role of the microenvironment. Curr Opin Cell Biol 2015; 36: 13-22.

14 Lambert AW, Pattabiraman DR, Weinberg RA. Emerging Biological Principles of Metastasis. Cell 2017; 168: 670-91.

15 Cockerell CJ, Wharton JR. New histopathological classification of actinic keratosis (incipient intraepidermal squamous cell carcinoma). J Drugs Dermatol 2005; 4: 462-7.

16 Saenz-Sarda X, Carrato C, Perez-Roca L et al. Epithelial-to-mesenchymal transition contributes to invasion in squamous cell carcinomas originated from actinic keratosis through the differentiated pathway, whereas proliferation plays a more significant role in the classical pathway. J Eur Acad Dermatol Venereol 2017.

17 Tao L, Huang G, Song $\mathrm{H}$ et al. Cancer associated fibroblasts: An essential role in the tumor microenvironment. Oncol Lett 2017; 14: 2611-20.

18 Glentis A, Oertle P, Mariani P et al. Cancer-associated fibroblasts induce metalloprotease-independent cancer cell invasion of the basement membrane. Nat Commun 2017; 8: 924.

19 Omland SH, Wettergren EE, Mourier T et al. Cancer associated fibroblasts (CAFs) are activated in cutaneous basal cell carcinoma and in the peritumoural skin. BMC Cancer 2017; 17: 675. 
TABLE LEGEND

Table1. Demographic and clinical characteristics $(\mathrm{N}=307)$

\begin{tabular}{lc}
\hline Characteristic & $\mathrm{n}(\%)$ \\
\hline Sex & \\
$\quad$ Male & $226(73.6)$ \\
$\quad$ Female & $81(26.4)$ \\
Age, years & $81(76-86)^{\dagger}$ \\
Patients with more than 1 iSCC in history & $183(59.6)$ \\
Tumour thickness & $1.9(1.1-3.9)^{\dagger}$ \\
Invasion depth (Clark level) & \\
into the papillary dermis (II) & $4(1.3)$ \\
to the junction of papillary and reticular dermis (III) & $31(10.1)$ \\
$\quad$ into the reticular dermis (IV) & $233(75.9)$ \\
into the subcutaneous fat (V) & $39(12.7)$ \\
Histological differentiation & \\
well differentiated & $262(85.3)$ \\
moderately differentiated & $43(14.0)$ \\
poorly differentiated & $2(0.7)$ \\
$\quad$ undifferentiated & $0(0)$ \\
Perineural invasion & $12(3.9)$ \\
Tumour sites & \\
scalp & \\
forehead & \\
parietal & \\
cheek & $62(27.7)$ \\
ear & $48(15.6)$ \\
nose & $48(15.6)$ \\
lip & $30(9.8)$ \\
& $22(7.2)$ \\
& $12(3.9)$ \\
&
\end{tabular}

iSCC, invasive squamous cell carcinoma

${ }^{\dagger}$ Data are median (IQR)

This article is protected by copyright. All rights reserved. 
Table 2. Histological grading and characteristics of adjacent and overlying epidermis to isCC

\begin{tabular}{lc}
\hline Characteristic & $\mathrm{n}(\%)$ \\
\hline Epidermis adjacent to iSCC & $8(2.6)$ \\
normal skin (no AK) & \\
AK histological severity & $161(52.4)$ \\
AK I & $117(38.1)$ \\
AK II & $21(6.8)$ \\
AK III & \\
AK basal growth grading & $9(2.9)$ \\
no basal growth & $79(25.7)$ \\
PRO I & $98(31.9)$ \\
PRO II & $121(39.4)$ \\
PRO III & $85(27.7)$ \\
\hline Epidermis overlying iSCC & $222(72.3)$ \\
Ulceration >50\% (no evaluation) & $76(34.2)^{\dagger}$ \\
AK histological severity & $69(31.1)^{\dagger}$ \\
AK I & $77(34.7)^{\dagger}$ \\
AK II & $73(32.9)^{\dagger}$ \\
AK III & $118 / 242(48.8)^{\ddagger}$
\end{tabular}

${ }^{t}$ referring to lesions eligible for evaluation of overlying epidermis $(N=222)$;

Fonly in 242 cases, adnexal structures were apparent and thus eligible for evaluation iSCC, invasive squamous cell carcinoma; AK, actinic keratosis

This article is protected by copyright. All rights reserved. 
(a)

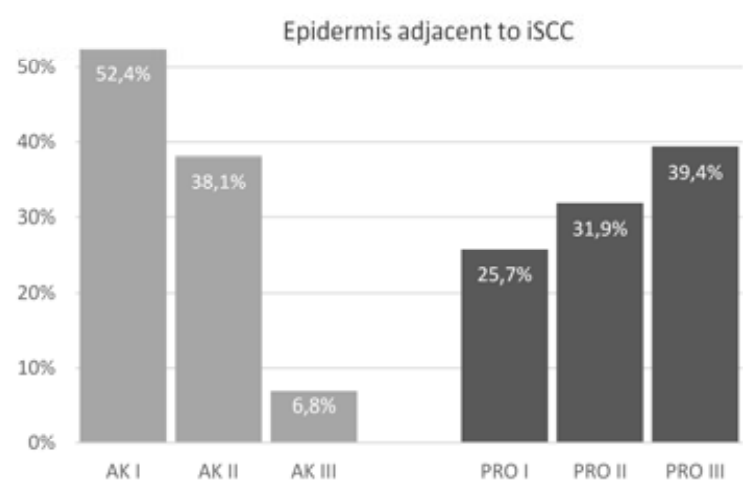

(b)

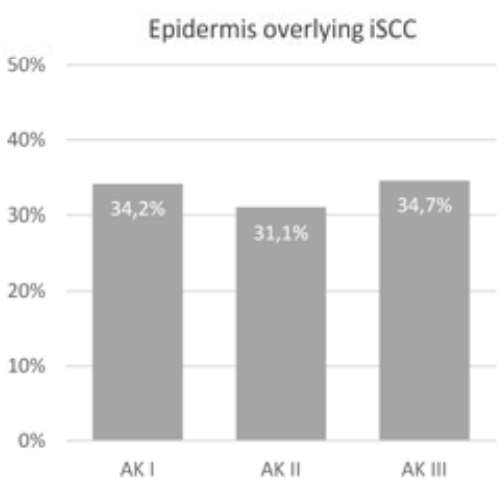

This article is protected by copyright. All rights reserved. 


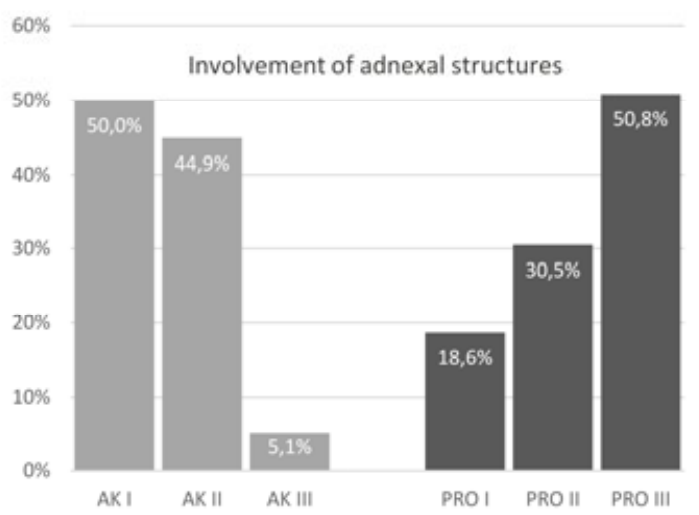

This article is protected by copyright. All rights reserved. 

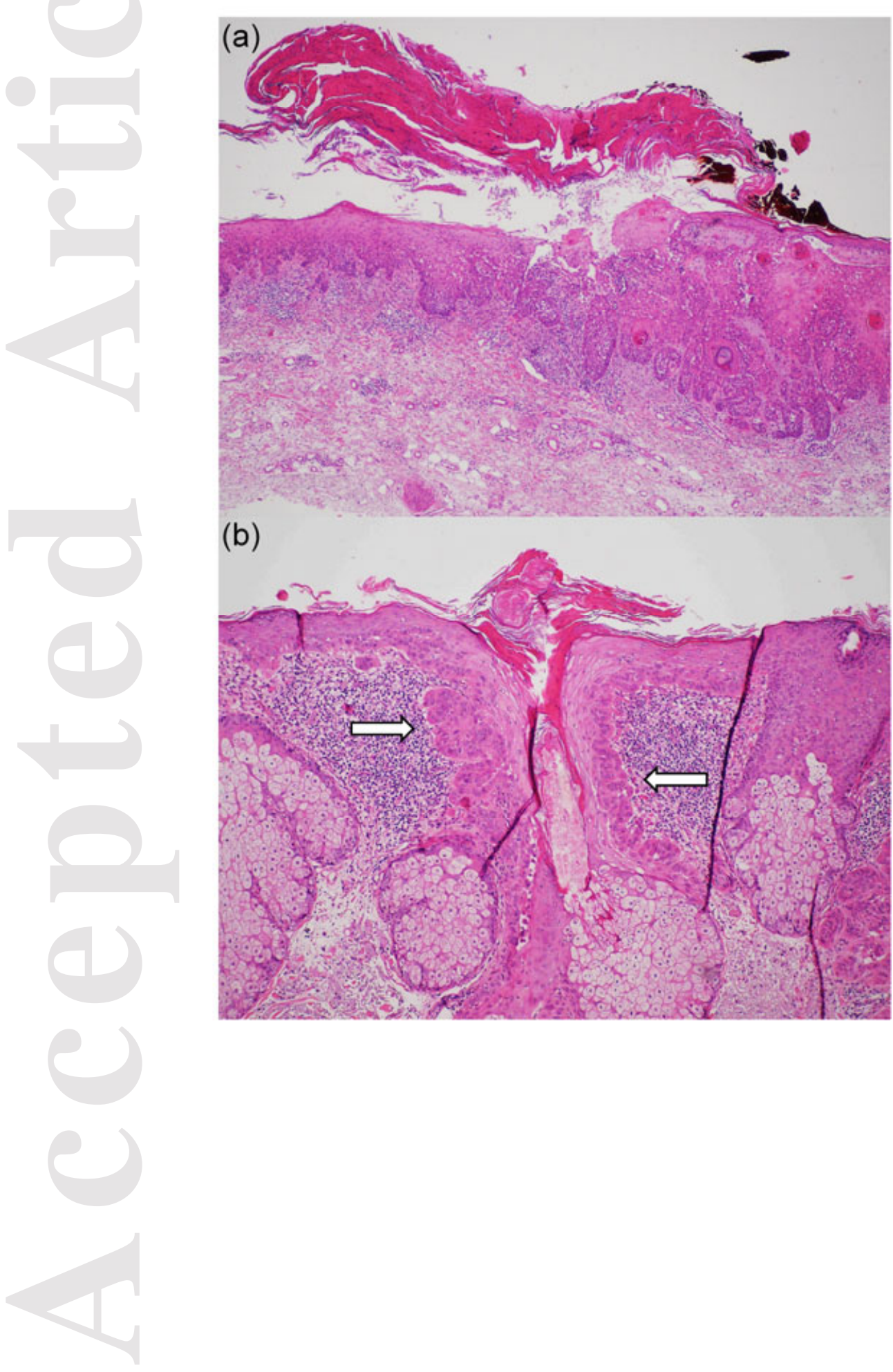

(b)

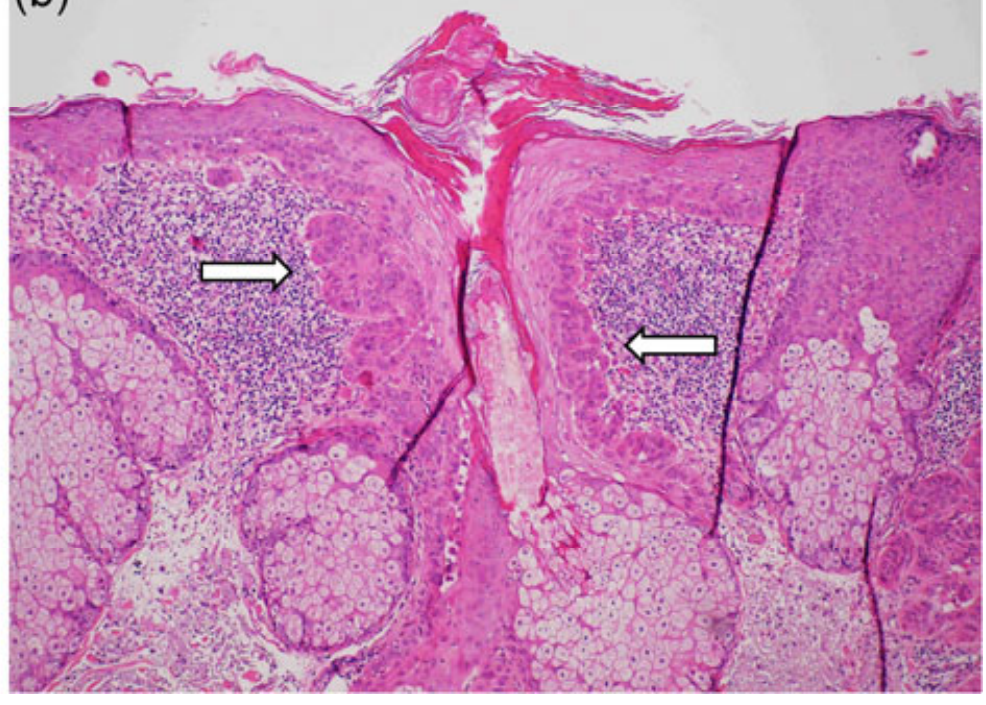

This article is protected by copyright. All rights reserved. 